\title{
DATA ANALYSIS METHOD SUPPORTING CAUSE AND EFFECT STUDIES IN PRODUCT-SERVICE SYSTEM DEVELOPMENT
}

\author{
J. Wall $ه$, O. K. Aeddula and T. Larsson \\ Blekinge Institute of Technology, Sweden \\ $\bigotimes$ johan.wall@bth.se
}

\begin{abstract}
A data analysis method aiming to support cause and effect analysis in design exploration studies is presented. The method clusters and aggregates effects of multiple design variables based on the structural hierarchy of the evaluated system. The resulting dataset is intended as input to a visualization construct based on colour-coding CAD models. The proposed method is exemplified in a case study showing that the predictive capability of the created, clustered, dataset is comparable to the original, unmodified, one.
\end{abstract}

Keywords: visualisation, product-service systems (PSS), product development, data analysis

\section{Introduction}

The act of designing industrial products or services is seldom done by one individual, rather it is normally a group effort (McComb et al., 2015). Collaboration in design can therefore be thought of as joint problem solving, i.e. working with others, sharing a common understanding of goals and available means, attempting to find solutions that are satisfying to all involved stakeholders. The knowledge needed to make well informed decisions rarely resides in single persons, not even departments. In contrast, cross-functional teams willing to share their knowledge and values are needed (Murakami, 2016). Wang et al. (2002) further emphasize the importance of cross-functional teams for rapid and reliable evaluation of different options in conceptual design. This development is fueled by the since long prevailing concurrent engineering approach in product development, and the transition towards product-service systems as well as increasing product complexity (Sundin et al., 2009).

As a consequence of the evolving product development landscape there is a significant need for collaboration, both internally within an organisation but also externally with suppliers, customers and other stakeholders. This is especially true in the early conceptual phase of product development where information is scarce and potentially conflicting. Hence, there is a clear incentive to involve all stakeholders into the decision process. Gathering the involved, normally diverse, group of stakeholders in a collaborative setting for design exploration exercises, sharing knowledge, values, and data is believed to augment decision making ability in early design. Research interest regarding an interactive group workspace focused on digital workflows has increased in recent years, see for example Nieminen et al. (2013) or Benyon and Mival (2015). Research regarding model-centric interactive workspaces are however not as common, an exception is the decision theatre at Arizona State University, see for example White et al. (2015). At Arizona State focus is on sustainability and how decisions affect the system in study on a macroscale. The need for model-centric environments in engineering design has 
been presented by Rhodes and Ross (2016). In line with that, Wall et al. (2018) proposes a model-driven environment for collaborative decision making focused on early phases of engineering design and presents initial work on conceptualizing, developing, and testing such an environment. Data analysis and visualisation become key enablers in these environments aiding information sharing, communication, understanding and building of knowledge within the cross-functional team (Wall et al., 2018).

Visualization may augment problem-solving capabilities by enabling the processing of more data without overloading the user. Cognitive tools propel users into far more effective thinkers and computer-based tools with visual interfaces may be the most powerful and flexible cognitive systems (Ware, 2005). Several authors have proposed visualisation constructs intended to support the product development process based on colour-coding the CAD model of the studied system. In colour-coding, system attributes are mapped to a colour scale to highlight components or subsystems that are negatively or positively affected by new designs, schematically exemplified in Figure 1. OstadAhmad-Ghorabi et al. (2009) discusses colour-coding of the CAD model to visualise the environmental impact of components based on a life cycle assessment. Bertoni (2013) developed a lifecycle value representation approach connecting qualitative value scores to the actual CAD representation of the product under analysis. Geromin et al. (2018) proposes to colour-code CAD models to visualize design rationale maturity. Described applications of colour-coded CAD models are either described on a conceptual basis or based on qualitative assessments of attributes to be visualized. However, for colour-coding to be viable in design exploration schemes based on automated simulation setups, methods supporting quantitative analysis linking attributes to specific components in the studied system is necessary.

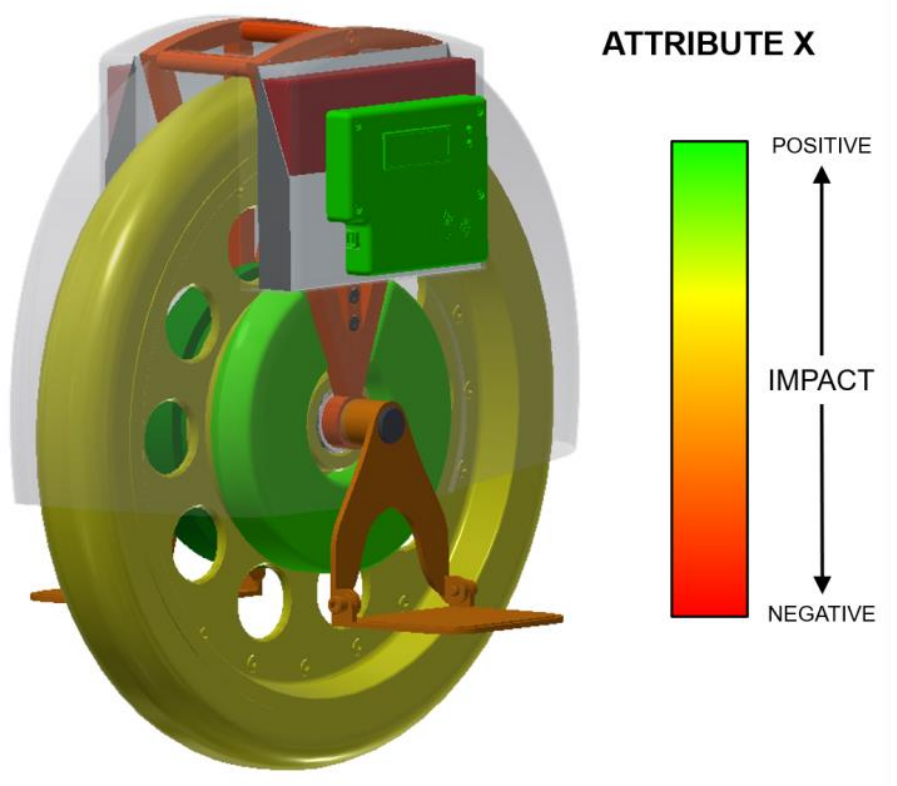

Figure 1. Example of colour-coded visualization

Based on the hypothesis that "Associating data and information with identifiable components and subsystems within the studied system is an efficient way to share information, aiding understanding in a cross-functional team" this paper presents a data analysis method enabling visualisation of quantified cause and effect relationships by colour-coding the CAD representation of the studied system. More specifically the aim is to develop a method able to support quantification of dependencies between design variables (independent variables) and design attributes (dependent variables) on a component or subsystem level in cases where more than one independent variable drives the configuration of the component or subsystem.

The remainder of this paper is organized as follows; In Section 2, the hierarchical system view is presented. Section 3 presents the proposed data analysis method. In section 4 the proposed method is exemplified through a case study. The paper concludes with discussion, conclusions and directions for future research. 


\section{System view and analysis}

Complexity of engineered systems has increased rapidly over the last decades going from essentially mechanical towards mechatronic systems that to a large extent relies on embedded software to fulfil functionality. Demands on a prospective solutions connectedness carried by the rapid development of Internet of Things combined with the conceivable development of communication and information technology further adds to this. There is no concise definition of what a complex system is (Ladyman et al., 2013). In the current work a complex system is defined by the integration of subsystems forming a system that exhibits behaviour not attainable by any of the subsystems alone. The interaction of the subsystem elements often results in emergent behaviour of the complex system that was not originally intended, designed, or desired. To be able to assess such system using a model-based approach, concept description needs to consider these interactions and component interdependencies according to overall system description. A common approach, attempting to make complex systems comprehensible, is to view them as a top-down hierarchy, decomposing the system down to indivisible parts. This decomposition is generally done from a structural point of view. In a structural hierarchy, components reside at the lowest level. At the mid-level, components are assembled into functional units, so-called sub-systems. At the top-level of the hierarchy, an assembly of sub-systems are connected to form a system able to perform a desired function. This way of visualizing systems makes them comprehensible for a wider audience within the development effort of the solution.

Information about a system might be conveyed using other hierarchical descriptions, for example the attribute hierarchy. An attribute can be defined as any aspect of the product itself or its use that can be used to compare product alternatives (Grunert, 1989). As an example, attributes of an automobile might be acceleration or fuel consumption. System attributes might be classified in numerous ways, see for example Crnkovic and Larsson (2004). In the current study attributes are divided into two types, directly composable and derived attributes. A directly composable attribute is a function of and only of the same attribute. Whereas a derived attribute is composed by other attributes of the system. As a derived attribute and the attributes it is derived from has a parent-child relationship, a hierarchy of attributes and associated quantifiable models used to predict them may be defined as exemplified in Figure 2. The structural and attribute hierarchies are directly interrelated as the structural hierarchy in combination with contextual information, such as usage scenarios, are the input to the models used to predict the attributes in the performance and resource space respectively.

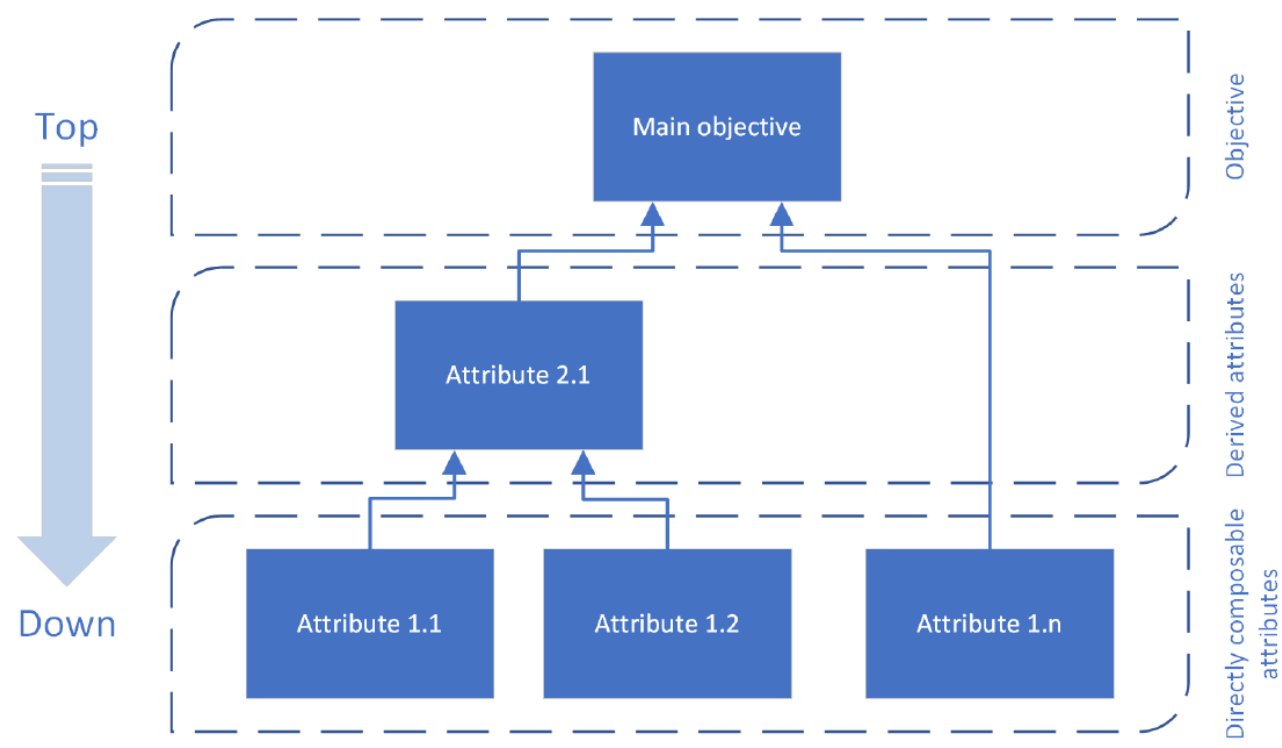

Figure 2. Attribute hierarchy

A hierarchical description of attributes might foster a shared understanding how these attributes are related. As such it might act as a boundary object (Larsson, 2003), mitigating negotiation regarding system design within the cross-functional team. However, to truly understand cause and effect relationships, i.e. how alternation of a proposed design concept affects a specific attribute or the 
understanding of how the concept should be modified in order to reach a specific target, experimentation is needed. By generating, and through computational methods evaluate and compare all feasible conceptual solutions in the current design space is rarely a practical solution. Furthermore, in an extreme case, if an optimal solution was stumbled upon early on in an exploration endeavour the ability to recognize that is lacking unless comparative studies are conducted. Commonly, datasets to be analysed origins from experiments varying independent variables according to a specified experimental plan, this is referred to as Design-of-Experiment (DOE). DOE is a technique for choosing a limited set of data samples in the design space with the goal of maximizing the amount of information produced (Giunta et al., 2003). When the experiment is executed, associated attributes for all concept variants in the DOE generated experimental plan are predicted and stored in a database. This type of design exploration activities in relation to complex systems may generate an abundance of data containing an intricate hierarchy of attributes. To understand how attributes on different levels is affected by system/subsystem/components or on a more detailed level relates to component features is imperative, making exploration of cause and effect relationships through data analysis a vital task.

The independent variables in a design exploration study might, for example, be associated with the proposed mechanical structure, usage scenario or even other attributes of the studied system. The resulting data structure of an experiment, including independent and dependent variables, is classified as a two-level variable hierarchy according to the left part of Figure 3. However, typically these independent variables are not identifiable as components or subsystems. Rather a subset of the independent variables in an experiment, combined with parameters and constants, drive the configuration of a particular component or sub-system and, as a consequence, also attributes associated with that component or sub-system. This might be visualized as an intermediate level in the variable hierarchy of the analysis as exemplified in the right part of Figure 3. As this intermediate level is not directly included in the conducted experiment, data to populate and in the next step analyse it, does not exist. To create this intermediate level, data may be synthesized by clustering the identified subset of independent variables and aggregate their effect on the dependent variable. To fit the intended visualisation scheme, by colour-coding the CAD model as discussed in the introduction, the independent variables are clustered based on the structural decomposition given by the structural hierarchy. A method for how to synthesis data for this intermediate level is described in section 3 .
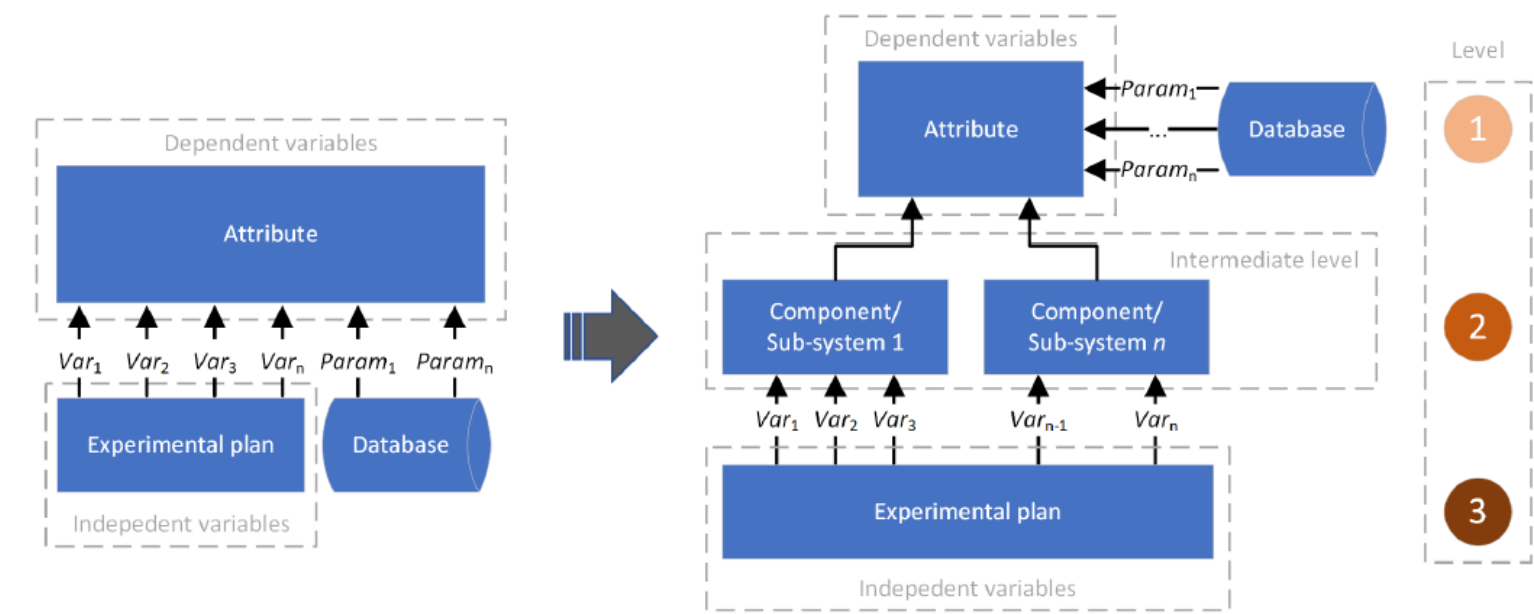

Figure 3. Variable hierarchy

\section{Data analysis method}

The cause and effect relationship between dependent and independent variables may be studied by establishing mathematical relationships between them and analysing the dependent variable dependencies on independent variables from the equation coefficients as is done in for example regression analysis (Allen, 1997). This type of analysis is well established studying relationships between dependent and independent variables that are directly observable. Generating new, clustered, 
variables that are inferred from the direct variables and establishing the relationship between clustered variables and the dependent variables are however challenging. A data analysis method is proposed to cluster the independent variables according to the structural hierarchy and construct a new intermediate level in the variable hierarchy. The method is designed to work under the assumptions that the variables are continuous and that the relationships between dependent variable and independent variables are linear. The assumption of linearity is a simplification applied in this work aiming at presenting an initial methodology for this type of analysis. Furthermore, the method is designed to analyse relationships between one dependent variable and several independent variables. Figure 4 depicts an overview of the proposed method, schematically also showing its role in the concept evaluation process. In the proposed method, data is partially regressed between dependent and independent variables and the respective independent variable coefficient is utilized to cluster the variables, creating an intermediate hierarchical level. All the variables are standardized to bring down the variables with different metric units to a single scale, to analyse the dependent variable dependencies on the independent variables (Bring, 1994). Standardization reduces the mean value of the variables to " 0 " and standard deviation to " 1 ", implying that all the variables are distributed normally on the same scale, which helps to understand which independent variables has greater effect on the dependent variable, when they have different metric units (Devore, 2012). Partial least square regression is applied again to understand the cause and effect relationships for the desired hierarchical levels or between desired dependent and independent variables.

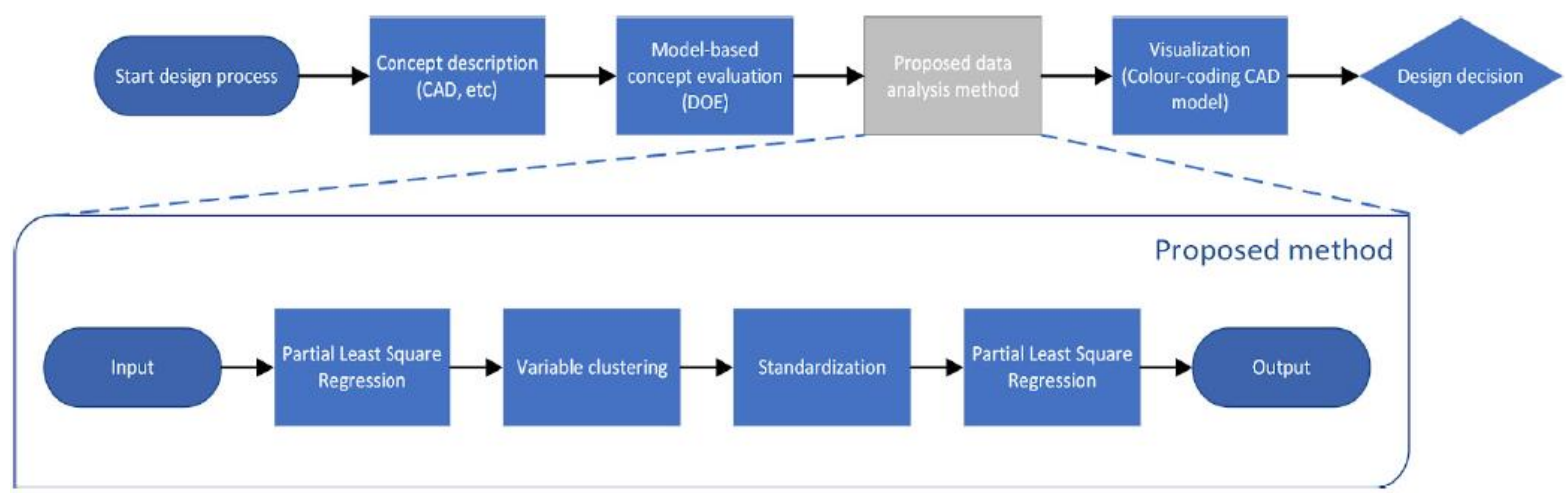

Figure 4. Overview of proposed method

\subsection{Partial regression}

Partial least square (PLS) regression is a statistical method to find the linear regression model for the desired input and output variables. It generates a regression model even when there are less number of concepts than number of variables (Rännar et al., 1995). The dataset consists of generated independent and dependent variable, where the dependencies are expressed in terms of an equation between them, there can be an infinite number of solutions satisfying the equation i.e., either nonparallel lines or identical lines or intersecting at a common point on independent variables plane. Using PLS, helps to identify the unique solution, which inter-links grouping variables and the output variable. PLS is useful to extract the variables that are not directly observed but are rather being inferred from other variables, in a way it assists to cluster the variables for a new hierarchical level (Pirouz, 2006).

In this context, partial least squares regression is used before standardization to understand the dependencies of the independent variables to be clustered and to generate an intermediate level in the variable hierarchy with respect to the dependent variable. In general, only a subset of existing independent variables are clustered and mapped onto the new intermediate level. The generated variables of the new hierarchy level are created with respect to the dependent variable to keep the contributions of the nonclustered independent variables being fixed with respect to the output at any hierarchy level. If the variables are clustered by dimension reduction method and without considering the dependent variable, then the significance of the contributions of the non-clustered variables are lost during the experimentation.

Dependency of dependent variable on the independent variables are analysed using the regression coefficients $(\beta)$. PLS finds the regression model by projecting the dependent variable $(Y)$ and independent variable matrix $(X)$ to another new space, finding the multidimensional direction in $X$ 
space that explains the maximum multidimensional variance direction in the $Y$ space. Considering the linear assumption between the dependent and independent variables, according to the Equation (1).

$$
Y=\beta X+C
$$

where $Y$ is $(n \times 1)$ matrix of the dependent variable and $X$ is $(n \times p)$ matrix of the independent variables, $C$ is the noise ( $p \times 1)$ matrix and $\beta$ is an $(n \times 1)$ matrix of regression coefficients. PLS decomposes the dependent and independent variables according to Equations (2) and (3) to maximise the covariance of $T$ in $X$ and $Y$ space (Boulesteix and Strimmer, 2006).

$$
\begin{aligned}
& Y=T Q^{T}+F \\
& X=T P^{T}+E
\end{aligned}
$$

where $T$ is $(n \times 1)$ matrix giving the latent components for the variable observations, $P(p \times 1)$ matrix and $Q(q \times 1)$ matrix are matrices of coefficients and $E(n \times p)$ and $F(n \times q)$ are random error terms. The latent component $T$ matrix is constructed as a linear function of $X$, as in Equation (4).

$$
T=X W
$$

where $W$ is $\left(\begin{array}{lll}p & x & 1\end{array}\right)$ matrix of weights. The latent components matrix $T$ is used to predict the regression coefficients and matrix of coefficients $Q . Q$ matrix is obtained as the least square solution of Equation (2).

$$
Q^{T}=\left(T^{T} T\right)^{-1} T^{T} Y
$$

$Q^{T}$ in Equation (2), transformed to Equation (6)

$$
\mathrm{Y}=(X W) Q^{T}+F
$$

Comparing Equation (6) and Equation (1) results in Equation (7)

$$
\beta=W Q^{T}
$$

Regression coefficients $(\beta)$ has a minimum norm satisfying the Equation (1)

$$
\text { Min }\|\beta\| \text { such that } Y=\beta X
$$

Where $\beta$ is calculated using the Equation (8)

$$
\beta=W\left(T^{T} T\right)^{-1} T^{T} Y
$$

The regression coefficients $(\beta)$ signifies how much the mean of the dependent variable changes when there is a one-unit shift of independent variable while keeping the others fixed. The sign represents the directional change, i.e., when there is a positive coefficient the dependent variable increases, as the particular independent variable increases and vice versa.

\subsection{Variable clustering}

Variable clustering involves the grouping of independent variables to form a new variable. Initial regression coefficients of the original independent variables are utilized to cluster the variables, according to Equation (9). In this work independent variables are clustered according to referenced structural hierarchy, independent variables belonging to the same component or subsystem are clustered together. Partial least square regression is applied with the dependent variable and independent variables to generate the regression coefficient matrix $\left[\beta_{0}, \beta_{i_{1}}, \beta_{i_{2}}, \ldots \ldots\right]$ and the respective regression coefficient is multiplied with the respective independent variable.

$$
X_{\text {new }}=\left(\beta_{0}+\beta_{i_{1}} x_{i_{1}}+\beta_{i_{2}} x_{i_{2}}+\cdots\right)
$$

where $X_{\text {new }}$ is new derived variable created by clustering sub-set of the independent variables. $\beta_{0}$ is the intercept term, $\beta_{i_{1}}$ the regression coefficient for $i_{1}$ independent variable and dependent variable and $x_{i_{1}}$ is the independent variable to be clustered. The regression coefficients are either negative or positive, when combined with the independent variables according to the Equation (9), it is most likely that there will be a positive regression coefficient for the clustered variable with the dependent variable, in-order to maintain the sign convention with the original independent variables. To avoid 
this issue, the individual effect of the variables in the clustered group is investigated, whether there is a larger negative regression coefficient or larger positive regression coefficient by summing up all the similar independent variables regression coefficient. If the summation is negative value, then Equation (9) is multiplied by -1 or else the Equation (9) is valid.

\section{Case study}

To exemplify the proposed method, it was used to analyse an existing dataset originating from a design exploration study evaluating proposed concepts of road construction equipment. In the study different design configurations for the vehicle platform were evaluated considering both performance and resource space. Concept evaluations were done through model-based experimentation (simulation). Simulation is an effective means to enable extensive exploration, so to learn faster (by performing more and earlier iterations) about the characteristics of the best possible design (see: Thomke and Fujimoto, 2000). A Value Driven Design (VDD) approach were applied as Systems Engineering (SE) research has stressed the importance of the value model to frontload engineering design activities (Collopy and Hollingsworth, 2011). This model is expressed as a single objective function that aims at measuring the "goodness" of the design.

In the study 700 variants of the vehicle platform was studied. Variations were driven by seven design variables (independent variables in the study) and 16 functional attributes were assessed. Based on these, derived attributes in resource space was assessed. More information on how this dataset is generated and the applied modelling and simulation scheme can be found in Bertoni et al. (2019). The value model was fed with the output of these models to render a value score for the design configuration under analysis. All aspects of value was quantified in monetary terms, enabling easy trade-off with more traditional requirements. This quantification process was based on the implementation of Net Present Value (NPV) from the VDD literature (Price et al., 2012). The dataset also contained information enabling mapping each independent variable directly to components or subsystems in the structural hierarchy.

The studied dataset contained three categorical variables rendering a non-linear behaviour. As proposed method is valid only for systems showing a linear behaviour, the dataset was linearized by fixing two out of three categorical variables to a specific choice and transforming the third one to continuous, making use of the dummy variables (Yip and Tsang, 2007). Dummy variables are numerical variables representing subgroups of the categorical variable. Number of subgroups for a categorical variable depends on the number of classes it contains. The model was custom trained to identify the type of categorical variable and regress accordingly. To exemplify the method, NPV was chosen as dependent variable, and the effect of the seven design variables were assessed. The regression coefficients for the dependent variable "NPV" are shown in the left part of Figure 5.
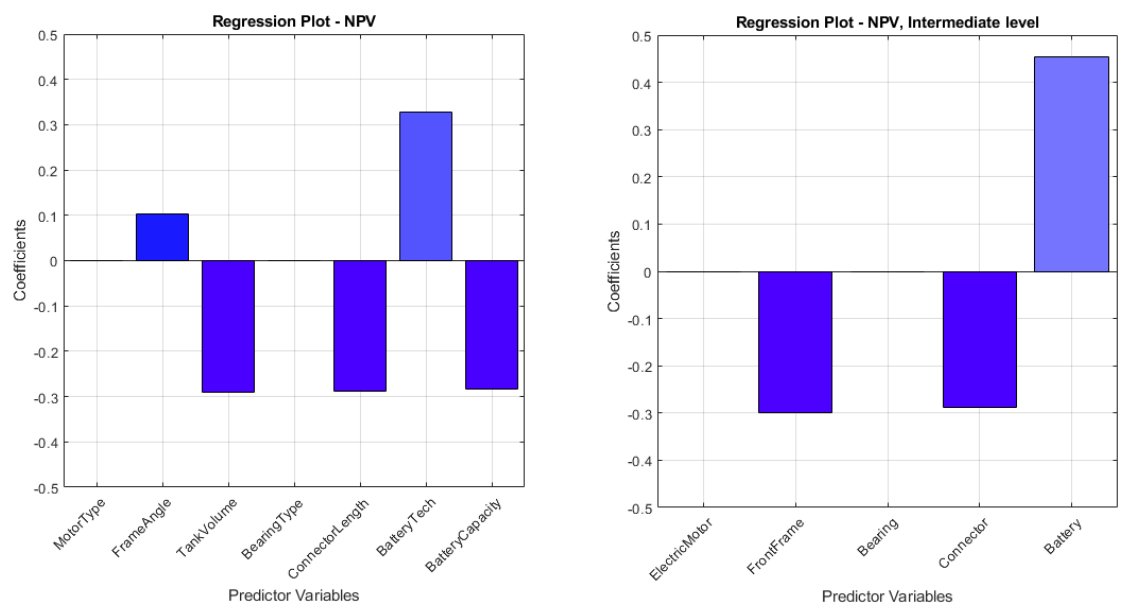

Figure 5. Regression Coefficients, example NPV

In the next step the independent variables were clustered according to the structural hierarchy, reducing them to five at the intermediate level of the variable hierarchy. These five variables are at this stage directly interpretable as components or subsystems of the studied system. The regression 
coefficients of the second stage are shown in the right part of Figure 5. In Figure 5, it can be seen that nor motor type or bearing type has an effect in this experiment. This is due to the applied linearization procedure where both these variables are set to fixed values.

Regression coefficients from the intermediate level are used to drive colour-coding of associated CAD-model. The data exchange between the simulation environment and the CAD application is enabled by establishing generic communication protocols/functions that exploits the application programming interface of each software. A colour scheme ranging from red to green over yellow is used. Red represents a negative effect on studied attribute whereas green represents a positive effect. Yellow indicates no or little effect on studied attribute. Figure 6 shows a mock-up of an intended implementation visualizing cause and effects within the attribute hierarchy. In this simplified example of visualisation, three out of the assed 16 attributes are shown as well as their aggregation in form of NPV rendered by the value model. Results from Figure 5 accompanied by results from similar results of the in the figure exemplified derived attributes, manoeuvrability, visibility and upgradeability, are shown. Components and subsystems not included in the experiment are shown as transparent.

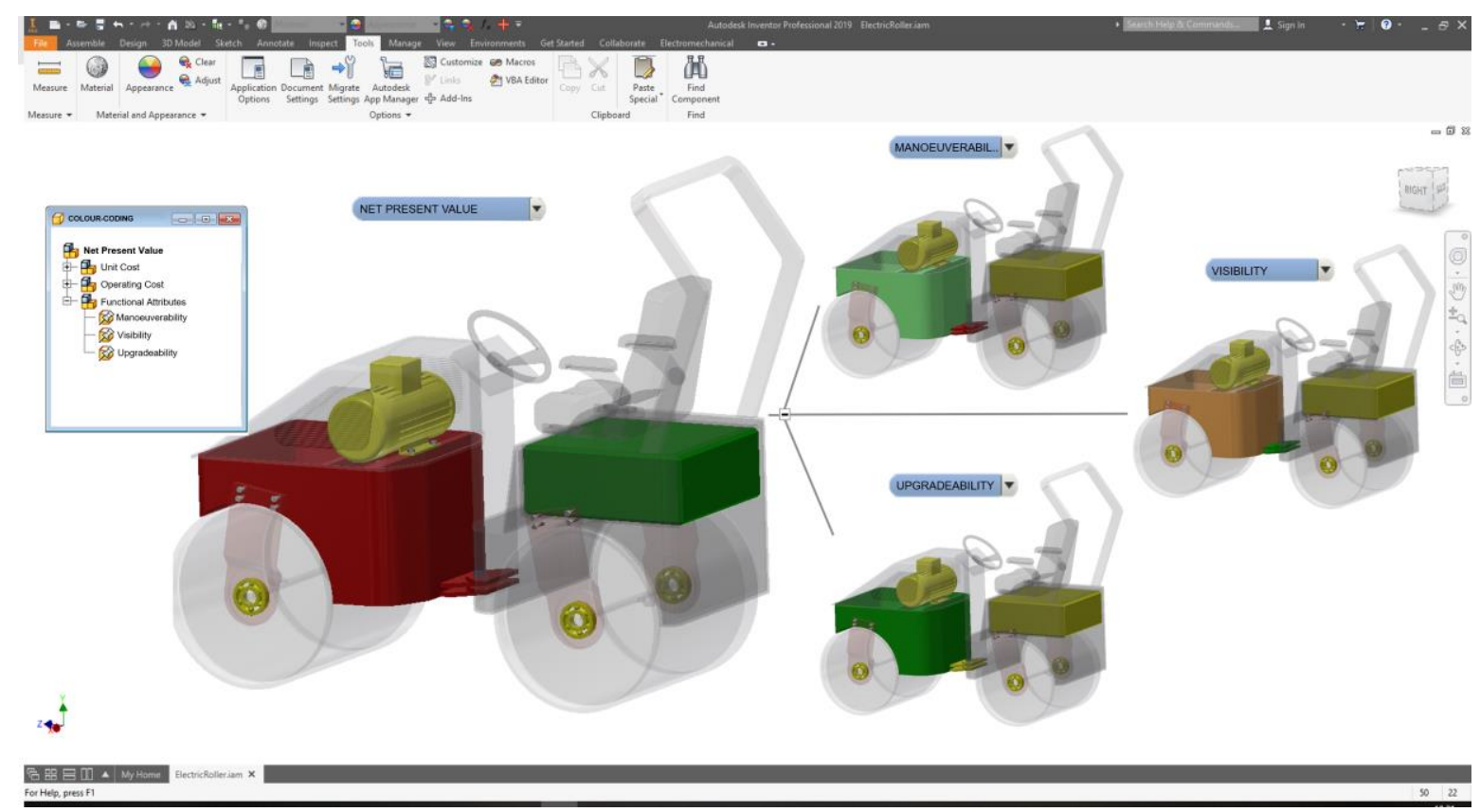

Figure 6. Colour-coded CAD-model in case study (CAD mock-up)

Coefficient of determination $\left(R^{2}\right)$ of a statistical model was used to validate the methodology. It determines how close the predicted data matches to the original data, according to the Equation (10) (Loretan and Kurz-Kim, 2007).

$$
R^{2}=1-\frac{\sum(y-Y)^{2}}{\sum(y-\bar{y})^{2}}
$$

where $y$ represents the actual data values, $Y$ is the predicted data values and $\bar{y}$ is the mean value of $\mathrm{y}$. The value of the regression coefficient of determination ranges from $[0,1]$. As the value of $R^{2}$ approaches unity, the predicted values are equal to the actual data values, i.e., the closer the value of $R^{2}$ to 1 , the greater is the fit of the data, and the closer the value of $R^{2}$ to 0 , the poorer is the fit of the data. For the NPV approximation, $R^{2}$ is 0.6402 , in comparison coefficient of determination of assessed derived attributes are 0.95 and above.

\section{Discussion and conclusion}

A data analysis method that clusters and aggregates effects of multiple design variables, independent variables in a study, is presented. The method aims to support cause and effect analysis supplying structured input to a visualisation construct. The intent is to map the analysed data based on the structural hierarchy of the proposed concept and to visualise the result using the CAD geometry as base. 
The proposed data analysis method is based on the partial least square regression method. The regression is conducted in two steps with an intermediate variable clustering through linkage to the proposed concept's structural hierarchy. The proposed method ensures that the significance of the contributions of the non-clustered variables are not lost during the experimentation. The proposed method has no limitation on number of variables it can handle. In practice however this will be an issue of availability of computational resources and time.

The method is exemplified through a case including visualisation by colour-coding the CAD model of the studied concept. Applying a validation scheme based on coefficient of determination analysis it is shown that the data structure using a "virtual" intermediate level performs, considering the non-linearities in the original dataset, comparably to the original dataset when it comes to predicting attributes.

As of now the application of the presented method is limited to problems where relationship between dependent and independent variables may be categorised as linear. During development of the algorithm datasets from multiple test cases were analysed. It was found that non-linear effects may affect the accuracy of the method to such an extent that, depending on the stage of the development project, these effects might need to be considered. Future work aims to extend the feasibility of the method to also include problems featuring non-linear relationships. An enhanced and generalized version of the proposed method, combining the data analysis and the visualisation construct, could be implemented as a design support in CAD software's enabling visualisation of quantified cause and effect relationships and thereby supporting collaborative decision making in design.

\section{Acknowledgement}

The research leading to these results has received financial support by the Swedish Knowledge and Competence Development Foundation (Stiftelsen för kunskaps- och kompetensutveckling) through the Model Driven Development and Decision Support research profile at Blekinge Institute of Technology.

\section{References}

Allen, M.P. (1997), Understanding regression analysis, Plenum Press, New York. https://doi.org/10.1007/b102242

Benyon D. and Mival, O. (2015), "Blended Spaces for Collaboration", Computer Supported Cooperative Work (CSCW), Vol. 24, pp. 223-249. https://doi.org/10.1007/s10606-015-9223-8

Bertoni, A. (2013), "Analyzing Product-Service Systems conceptual design: The effect of color-coded 3D representation”, Design Studies, Vol. 34 No. 6, pp. 763-793. https://doi.org/10.1016/j.destud.2013.02.003

Bertoni, M. et al. (2019) "Life cycle simulation to support cross-disciplinary decision making in early PSS design”, Procedia, ISSN 2212 - 8271, EISSN 83, Procedia CIRP, Elsevier. https://doi.org/10.1016/j.procir. 2019.03.138

Boulesteix, A.-L. and Strimmer, K. (2006), "Partial least squares: a versatile tool for the analysis of highdimensional genomic data", Briefings in Bioinformatics, Vol. 8 No. 1, pp. 32-44. https://doi.org/10.1093/ bib/bbl016

Bring, J. (1994) "How to Standardize Regression Coefficients", The American Statistician, Vol. 48 No. 3, pp. 209-213. https://doi.org/10.1080/00031305.1994.10476059

Collopy, P.D. and Hollingsworth, P.M. (2011), "Value-driven design”, Journal of Aircraft, Vol. 48 No. 3 , pp. 749-759. https://doi.org/10.2514/1.C000311

Crnkovic, I. and Larsson, M. (2004), "Classification of quality attributes for predictability in component-based systems", Proc. of Workshop on Architecting Dependable System, IEEE

Devore, J.L. (2012), Probability and statistics for engineering and the sciences, 8th ed., Brooks/Cole, Cengage Learning, Australia.

Geromin, A. et al. (2018), "CAD modelling based on knowledge synthesis for design rational”, Procedia CIRP, Vol. 70, pp. 156-161. https://doi.org/10.1016/j.procir.2018.01.008.

Giunta, A.A., Wojtkiewicz, S.F. and Eldred, M.S. (2003), "Oveview of modern design of experiment methods for computational simulation”, 41st Aerospace Sciences Meeting and Exhibit, Reno, Nevada. https://doi.org/10. 2514/6.2003-649

Grunert, K.G. (1989), “Attributes, attribute values and their characteristics: A unifying approach and an example involving a complex household investment”, In Journal of Economic Psychology, Vol. 10 No. 2, pp. 229251. https://doi.org/10.1016/0167-4870(89)90021-4

Ladyman, J., Lambert, J. and Wiesner, K. (2013) "What is a complex system?", European Journal for Philosophy of Science, Vol. 3 No. 1, pp. 33-67. https://dx.doi.org/10.1007/s13194-012-0056-8. 
Larsson, A. (2003) "Making sense of collaboration: the challenge of thinking together in global design teams", in. ACM Press, p. 153. https://dx.doi.org/10.1145/958160.958184.

Loretan, M.S. and Kurz-Kim, J.-R. (2007), "A note on the coefficient of determination in regression models with infinite-variance variables", Discussion Paper Series 1: Economic Studies. Available at: https://ideas.repec. org/p/zbw/bubdp1/5574.html (Accessed: 1 November 2019).

McComb, C., Cagan, J. and Kotovsky, K. (2015), "Rolling with the punches: An examination of team performance in a design task subject to drastic changes", Design Studies, Vol. 36, pp. 99-121. https://doi.org/10.1016/j.destud.2014.10.001

Murakami, Y. (2016), "Linking knowledge absorption and transmission toward innovation in R \& D organizations", in Proceedings of the 17th European Conference on Knowledge Management, ECKM 2016. vol. 2016-January, Academic Conferences Limited, pp. 667-675.

Nieminen, M.P., Tyllinen, M. and Runonen, M. (2013), "Digital war room for design", Proceedings of the 15th international conference on Human Interface and the Management of Information: information and interaction for learning, culture, collaboration and business, July 21-26, Las Vegas, NV. https://doi.org/ 10.1007/978-3-642-39226-9 39

Ostad-Ahmad-Ghorabi, H., Collado-Ruiz, D. and Wimmer, W. (2009), "Towards Integrating LCA into CAD", Proceedings of ICED 09, the 17th International Conference on Engineering Design, Vol. 7, Design for X I Design to X, Palo Alto, CA, USA, pp. 301-310.

Pirouz, D.M. (2006), “An Overview of Partial Least Squares”, SSRN Electronic Journal. https://doi.org/ $10.2139 / \mathrm{ssrn} .1631359$

Price, M. et al. (2012), "A novel method to enable trade-offs across the whole product life of an aircraft using value driven design", Journal of Aerospace Operations, Vol. 1 No. 4, pp. 359-375. https://doi.org/10.3233/ AOP-120028

Rhodes, D.H. and Ross, A.M. (2016), "A vision for human-model interaction in interactive model-centric systems engineering”, In 26th Annual INCOSE International Symposium, Edinburgh. Scotland. https://doi.org/10.1002/inst.12162

Rännar, S. et al. (1995), “A PLS kernel algorithm for data sets with many variables and few objects. Part II: Cross-validation, missing data and examples", Journal of Chemometrics, Vol. 9 No. 6, pp. 459-470. https://doi.org/10.1002/cem.1180090604

Sundin, E. et al. (2009), "Challenges for Industrial Product/Service Systems - Experiences from a learning network of large companies", In: Industrial product- service systems (IPS2): proceedings of the 1st CIRP IPS2 Conference, 01 - 02 April, Cranfield University, UK.

Thomke, S. and Fujimoto, T. (2000), "The effect of "Front-Loading“" problem-solving on product development performance", Journal of Product Innovation Management, Vol. 17 No. 2, pp. 128-142. https://doi.org/ 10.1016/S0737-6782(99)00031-4

Wall, J., Bertoni, M., and Larsson T. (2018), “A model-driven decision arena: Augmenting decision making in early design”, Proceedings of NordDesign 2018, August 16th-18th Linköping.

Wang, L. et al. (2002), "Collaborative conceptual design—state of the art and future trends", Computer-Aided Design, Vol. 34 No. 13, pp. 981-996. https://doi.org/10.1016/S0010-4485(01)00157-9

Ware C. (2005), "Visual Queries: The Foundation of Visual Thinking”, In: Tergan, S.O. and Keller, T. (eds), Knowledge and Information Visualization. Lecture Notes in Computer Science, Vol. 3426, Springer, Berlin, Heidelberg. https://doi.org/10.1007/11510154_2

White, D.D. et al. (2015), “Water management decision makers' evaluations of uncertainty in a decision support system: the case of WaterSim in the Decision Theater", Journal of Environmental Planning and Management, Vol. 58 No. 4, pp. 616-630, https://doi.org/10.1080/09640568.2013.875892

Yip, P.S.L. and Tsang, E.W.K. (2007), "Interpreting dummy variables and their interaction effects in strategy research”, Strategic Organization, Vol. 5 No. 1, pp. 13-30. https://doi.org/10.1177/1476127006073512. 